\title{
Face Detection on Pre-modern Japanese Artworks using R-CNN and Image Patching for Semi-Automatic Annotation
}

\author{
Alexis Mermet \\ Ecole Polytechnique Fédérale de Lausanne (EPFL) \\ Lausanne, Switzerland \\ Chikahiko Suzuki \\ ROIS-DS Center for Open Data in the Humanities, \\ National Institute of Informatic (NII) \\ Tokyo, Japan
}

\begin{abstract}
We propose a face detection method for semi-automatic annotation of faces on pre-modern Japanese artworks to assist art historians identify objects in the art collection. Our method is based on R-CNN, such as Faster R-CNN and Cascade R-CNN, for object detection, and image patching for taking advantage of high resolution images. Our face detectors were first trained on the KaoKore dataset to demonstrate that existing object detection models with image patching can successfully learn faces in artworks. Our face detectors were then applied to the Kouhon dataset to assist art historians create a new facial expression dataset. Finally the impact of face detection on art history research was measured by the reduction of annotation time, and it was estimated to be $1 / 3$ in comparison to fully manual annotation.
\end{abstract}

\section{KEYWORDS}

Art collection, R-CNN, Face Detection, Image Patching, Semi-Automatic Annotation

\section{ACM Reference Format:}

Alexis Mermet, Asanobu Kitamoto, Chikahiko Suzuki, and Akira Takagishi. 2020. Face Detection on Pre-modern Japanese Artworks using R-CNN and Image Patching for Semi-Automatic Annotation. In 2nd Workshop on Structuring and Understanding of Multimedia heritAge Contents (SUMAC'20), October 12, 2020, Seattle, WA, USA. ACM, New York, NY, USA, 9 pages. https://doi.org/10.1145/3423323.3423412

\section{INTRODUCTION}

This paper proposes an automated face detection method on premodern Japanese artworks to reduce the cost of creating object-level annotation by art historians. First, an object detection method is applied to Japanese artworks to extract the bounding box of all faces in the image. Second, art historians annotate the content of the bounding box with appropriate metadata. Our work is based on collaboration between computer scientists and art historians who

Permission to make digital or hard copies of part or all of this work for personal or classroom use is granted without fee provided that copies are not made or distributed for profit or commercial advantage and that copies bear this notice and the full citation on the first page. Copyrights for third-party components of this work must be honored.

For all other uses, contact the owner/author(s)

SUMAC'20, October 12, 2020, Seattle, WA, USA

(C) 2020 Copyright held by the owner/author(s)

ACM ISBN 978-1-4503-8155-0/20/10.

https://doi.org/10.1145/3423323.3423412

\author{
Asanobu Kitamoto \\ ROIS-DS Center for Open Data in the Humanities, \\ National Institute of Informatic (NII) \\ Tokyo, Japan \\ Akira Takagishi \\ University of Tokyo \\ Tokyo, Japan
}

are working on style comparative study and are analyzing objects in the image to answer research questions such as identifying the date of production and the skill of creators. In their research, extracting objects from the image and creating the annotated database of objects is a fundamental task to answer research questions.

Our research group is especially interested in a particular type of objects, namely faces in pre-modern Japanese artworks. Hence our goal is to design an object detector for faces in art collections, which have different image features from natural scenes. As a starting point, we used the KaoKore dataset [15], which is an open dataset of facial expressions from pre-modern Japanese artworks manually created by art historians. It contains 8573 faces extracted from 1470 high quality images of pre-modern Japanese picture scrolls and picture books with annotated metadata. This dataset was used to train several face detection models for performance comparison, and the selected model was applied to a new artwork collection to help art historians create a new dataset for facial expressions.

\section{RELATED WORK}

This paper focuses on object detection, which is about identifying objects in a given image with the coordinates of a bounding box. Object detection methods using convolutional neural network are already well established. Standard methods, such as Single Shot Detector (SSD) [6], Faster R-CNN [9] and Cascade R-CNN [2], are often implemented using standard classification backbones such as VGG16 [12] and ResNet [5]. Object detection is usually applied to photographs or videos of natural scenes, where millions of images are available as training data. On the other hand, object detection for art collection has not been deeply studied, partly because training data is not abundant (e.g. 1470 images and 8573 faces in the KaoKore dataset). Deep learning was applied to find objects in artworks [14], but the purpose of the research was to assign image-level labels without estimating bounding boxes of objects in the image. Extracting the bounding box of faces using Fast R-CNN on the PeopleArt collection, a collection of people in the various styles of paintings even including abstract art, was also studied [16], but due to the challenging goal of the problem, the performance was relatively low, such as average precision of $50 \%$. Object detection were also applied to Japanese old books using Faster R-CNN [13], and the most similar problem setting to this paper was studied for a manga collection [8], in which the authors used bounding box 
annotations on manga images for training a variant of SSD300 as a face detector.

Based on the results of related work, it is clear that the usage of Faster R-CNN or SSD300 is a common choice for object detection on artworks, such as the face. Our contribution is two-fold. The first contribution is that we go beyond just evaluating the performance of the models on the dataset prepared in advance. Our trained model was applied to a completely new image collection without annotation at the time of training, and the generalization of the model was evaluated by comprehensively annotating a new dataset to obtain the precision and recall in a realistic setting, as described in Section 5.1. The second contribution is in the design of a machine learning pipeline for the face detector to work effectively on Japanese artwork collections. In particular, we focus on the problem of high image resolution because the reduction of image resolution leads to the deterioration of performance. We propose a method called image patching, as explained in Section 3.3. The method first divides a high resolution image into small patches with image padding, then applies the object detection model for each patch, and finally combines all detection results with NonMaximum-Suppression. Our approach is inspired by a work for style detection using a two-stage deep learning approach [11], in which a full image is divided into five rectangular patches so that each patch overlaps with four other patches at $25 \%$. This simple approach was generalized in this paper to solve detection problems by allowing more number of patches with any size using image padding.

Note that this paper focuses on style comparative study in art history research, which is based on the visual comparison of characteristics in artworks. The target of comparison, however, has a wide range of diversity, from comparison within the global art history to a more focused comparison for each genre or each painter group. Based on global artwork datasets, such as WikiArt, deep learning can be used for author identification [1], character identification [7] and style classification [4]. Our research, on the other hand, is motivated by more specific research questions, so the choice of methods and priority of research depends on the questions we want to solve. Based on the opinions from art historians, face identification is not realistic for most of the faces. and face detection should be prioritized than face classification. The organization of the paper reflects this choice.

\section{METHODS}

\subsection{Data splitting}

As said previously in Section 1, our work started from the KaoKore dataset, but the actual dataset we used for our training is a slightly modified version of it. Because our goal is to create a face detector, what we need are not cropped face images but full images containing pixels inside and outside of the bounding boxes. We took advantage of IIIF (International Image Interoperability Framework) to download full images from the image provider, and use them for our research.

Our data consists of artworks that originate from multiple picture scrolls and picture books. There is a large variations across artworks in the dataset, because each scroll or book has its own style. Therefore, we should not consider that faces in the KaoKore dataset are samples from the same distribution, but rather each scroll or book has its own distribution. In this case, a simple random split into training and testing sets is not appropriate for evaluating the quality of machine learning models. Instead, we define the three types of data splitting into training and testing sets:

Random split With this approach, $80 \%$ of the available images are randomly chosen to be in the training set and the $20 \%$ left are used for testing purposes only. 1176 images are used for training (corresponding to a total of 6866 faces) and 294 for testing (corresponding to a total of 1706 faces).

Inter-books split With this approach, we consider that images obtained from the same book must be in the same split of the data. This means that either the full book and the corresponding images are available for training or they are available for testing only. We randomly chose $80 \%$ of the books available as training materials. This corresponds to 76 of the 96 books from which data originate in our dataset. In total, we have 1236 training images (6998 faces) and 234 testing images (1574 faces).

Intra-books split With this approach, we consider that all the books are available for training. Then, in each book, we select $80 \%$ of the available images as training data and the $20 \%$ left are used for testing. If we want to formulate it differently, each book in the dataset is such that $80 \%$ of its content is available for training and the rest is kept for testing. In total, we have 1137 training images (6692 faces) and 333 (1880 faces) testing images.

Each split reflects its own problem setting, so performance for each split should not be used to compare which splits are "better" than others. We trained face detectors for each split and checked the performance to know about the effect of split types (see Section 4.1).

\subsection{Deep learning architectures for object detection}

As presented in Section 2, object detection is about estimating the bounding box of an object of interest in an image. We used object detection for face detection to estimate the bounding box of faces in pre-modern Japanese artworks. Our goal is to assess the performance of existing detectors on our dataset. The architectures we assessed are the following:

(1) SSD300[6]

(2) Faster R-CNN[9]

(3) cascade R-CNN[2]

Faster R-CNN and Cascade R-CNN detectors both aim at training an object detector for images with different aspect ratios during the training phase. Faster R-CNN does so by using Region Proposal Network (RPN) sharing features with the detector, while Cascade $\mathrm{R}-\mathrm{CNN}$ consists of a cascade of detectors with increasing quality. On the other hand, SSD is a simple convolutional neural networks in which some of its intermediate feature maps are used to detect objects of different sizes. All these models were setup using a ResNet50[5] backbone pre-trained on the ImageNet dataset to take advantage of transfer learning. Section 4 shows how these models were tested with our approach in multiple setups. 
Machine learning models for semantic segmentation or instance segmentation, such as Masked Cascade R-CNN, can also be applied to our task as long as we have appropriate training data. In the context of art history research, bounding box is a reasonable choice when the machine takes a role of localizing objects and the human expert, of interpreting objects. Pixel-level segmentation, on the other hand, is potentially advantageous when the machine takes both roles of localization and interpretation but we leave it for future work.

\subsection{Image patching}

The goal of image patching is to allow a high resolution input image to be passed to an object detection architecture without losing details due to resizing, because faces in big or long painting scrolls are sometimes too small to be detected from resized images. However, due to the limitation of the image size acceptable by an object detection architecture, reshaping of the input image should be done before entering the object detector. To solve this problem, we propose a method of image patching, inspired by style detection research as introduced in Section 2. Algorithm 1 illustrates a pseudocode of the image patching algorithm used for the detection phase.

Data: List of Japanese artworks I, and patching size s

Result: Bounding boxes for the faces contained in each artwork

for image $i$ in I do

Pad image i according to patching size s;

Crop all patches of size s from image $i$;

Add these patches to the list of patches, $\mathrm{P}$;

for patch $p$ in $P$ do

Detect faces in patch $\mathrm{p}$;

Add found faces to the list of found faces F; end

Translate faces in F into i's coordinate system;

Non-Maximum-Suppression to remove overlapping bounding boxes;

end

Algorithm 1: Pseudo-code of the image patching algorithm used for the detection phase.

3.3.1 Image patching during the training phase. During the training phase, each training image is split into multiple rectangular patches of the same size. In this experiment, these patches have the same size with the input size of the detector to avoid any resizing. Note that when the input size of the detector is too small for patches to contain entire faces, we use larger patches with the same aspect ratio for best resizing. To do so, each image is padded beforehand with the color of its border so that it can be split exactly with the desired patch size. The detector is then trained on the patches containing bounding boxes, which we call positive samples, to avoid forcing the model to find something from negative samples. The process looks like the following: In this schema we consider sequential patches and overlapping ones in the middle. We do this to avoid missing any faces in the images while multiplying potential

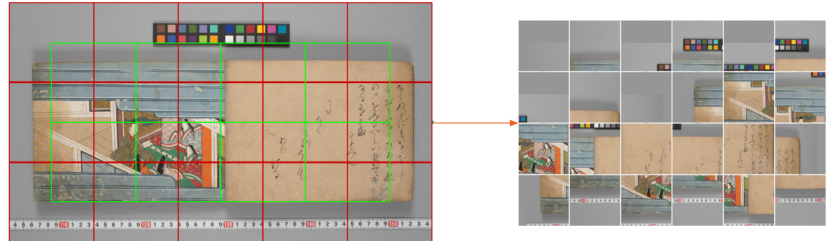

Figure 1: Schema of our patching procedure. On the left panel, we can find the full image with patches of size $600 *$ 600 drawn in colors. The red patches correspond to the sequential patches while the green ones are the overlapping patches. In the right panel, we can see all the obtained patches. Source: "KaoKore Dataset."

positions of faces. Each overlapping patch overlaps by $25 \%$ with four other sequential patches. As highlighted in the right-hand part of this schema, however, only a few of these patches can in fact be used for training; the ones containing faces.

The next step is to choose positive samples. To check if a positive sample contains a relevant part of the original bounding box, we focus on two aspects. First, a bounding box should contain facial pixels rather than background pixels. Second, a bounding box should contain an essential part of a facial expression, not just a part of the face, such as ear. These two aspects cannot be measured directly, so we instead used the Jaccard overlap measure.

The Jaccard overlap (IoU) is a measure of the extent to which two boxes overlap. The closer the value is to 1 , the more likely two boxes are overlapping. When this value gets closer to 0 , the two boxes are likely to represent mutually exclusive spaces.

$$
\mathrm{IoU}=A \cap B / A \cup B
$$

We set an IoU threshold $\theta$ on the Jaccard overlap between the true bounding box and the one contained in the patch. If the bounding box in the patch has a Jaccard overlap larger than $\theta$, the face is contained in the patch. Otherwise it is not.

3.3.2 Image patching during the testing phase. During the testing and detection phase, we apply a process similar to what we do for the training phase. Given an image with faces, we pad it and create multiple patches of the same size used for the training phase. For each of these patches, we detect faces and obtain multiple bounding boxes and their corresponding detection scores for each patch. These bounding boxes are then put back together and analyzed for duplication to obtain the final detection result on the original image.

To do so, we first convert the bounding box coordinates obtained for each patch to their coordinates in the original image. We now have a list of bounding boxes, potentially overlapping, and their detection score. Note that, at this stage, we keep only bounding boxes with a prediction score above a certain threshold, $\phi$. We then apply Non-Maximum-Suppression, inspired by the SSD model [6], to remove redundant predictions while keeping the ones with the highest prediction scores. Two boxes are judged overlapping when IoU is more than the threshold $\gamma$. 


\subsection{Evaluation metrics}

To assess the quality of the result, we use the standard definition of True Positive $t p$, True Negative $t n$, False Positive $f p$ and False Negative $f n$ to compute evaluation metrics as follows.

Recall is the number of properly retrieved element among all the elements that should have been well retrieved.

$$
\text { recall }=\frac{t p}{(t p+f n)}
$$

Precision is the number of properly retrieved element among all the elements that have been retrieved.

$$
\text { precision }=\frac{t p}{(t p+f p)}
$$

Mean Average Precision , abridged to mAP, is a measure falling between 0 and 1 used to compute the accuracy of an object detector at a given IoU level. This level corresponds to the threshold at which we consider that a predicted bounding box and a target bounding box are overlapping (see Jaccard Overlap in Section 3.3). The mean Average Precision of a model corresponds to the area beneath the precision-recall curve:

$$
m A P=\int_{0}^{1} p(r) d r
$$

At each level of the curve the precision $p$ for recall $r$ can be interpolated so that the computations are simplified:

$$
p_{\text {interp }}(r)=\max _{\widetilde{r}>r} p(\widetilde{r}) \text { and } m A P=\sum_{n}\left(r_{n+1}-r_{n}\right) p_{\text {interp }}\left(r_{n+1}\right)
$$

\section{EXPERIMENTS}

We first designed experiments on the KaoKore dataset to evaluate our proposed face detectors in terms of data splitting, deep learning models, and image patching.

In the following experiments, thresholds introduced in section 3.3 were set to fixed values as $\phi=0.5$ and $\gamma=0.3$, while $\theta$ was set to 0.4 , except for the experiment in Section 4.3. In terms of data splits, we used the same intra-books split, except for the experiment in Section 4.1. Our face detectors are developed using the mmdetection[3] library that offers an easy framework to setup multiple PyTorch object detection networks using Quadro RTX 5000 graphic cards.

Data augmentation also plays an important role in our experiments. In the case of SSD300, input images for training are first applied photometric distortion by randomly changing their brightness, contrast and saturation and by randomly swapping their color channels. Images are then randomly expanded to another aspect ratio or can be randomly cropped. These images are finally resized to a $300 * 300$ shape and passed to the detector while being normalized according to both the mean and standard deviation of the ImageNet dataset, and being randomly flipped. On the other hand, during the testing phase, input images are only resized and normalized. In the case of Faster R-CNN and Cascade R-CNN; input images are just resized to a $1333 * 800$ shape, shape corresponding to how the input layers of the two architectures are implemented in the library, in both the training and testing phase, normalized according to both the mean and standard deviation of the ImageNet dataset that was used during pre-training and randomly flipped.

\subsection{Face detection on the KaoKore dataset}

First we trained a basic object detector, SSD300 [6] with image patching, to check the feasibility of face detection on the KaoKore dataset. The experiment also checks the effect of three split types. For each split setups, we made 5 independent runs with different training and testing sets. The models were trained for 24 epochs with a SGD optimizer. The learning rate was set to $1 e-4$ at the start and changed at the 16th and 22nd iteration. The weight decay was set to $5 e-4$ and the momentum to 0.9 .

\begin{tabular}{|l|l|l|l|}
\hline Split type & Random & Inter-books & Intra-books \\
\hline mAP (\%) & 71.2 & 70.3 & 73.5 \\
\hline
\end{tabular}

Table 1: Result of mAP by an SSD300 detector. $\mathrm{mAP}$ is averaged for 5 independent runs on different data splits for Random split, Inter-books split and Intra-books split.

The result of average mAP scores is summarized in Table 1. We have the following observations from the result:

- We confirmed that machine learning-based face detectors work good for the KaoKore dataset, or for pre-modern Japanese artworks, which is the target of our research.

- Similar performance between random split and inter-books split shows that our model can generalize to books with different style, materials, color palettes and shapes from the training data.

- The best score of intra-books split shows that our model, when able to train on any available type of books (any type of images colors, shape, style, materials, etc...), is able to well detect faces on any images of our data-set.

The results obtained on the inter-books split are the more relevant to this work. They highlight that we can use our method on never seen books and scrolls and thus our approach could work for annotating a new collection, as we will do in Section 5.1. On the other hand, we run our experiments on the intra-books split for the rest of this study because it allows us to train our detectors on as many types of books as possible before testing our approach on a new collection.

\subsection{Comparison of architectures and image patching}

Next we compare the performance of three architectures as presented in Section 3.2, and the effect of image patching method. The baseline is the result without image patching for each architecture, and we also ran experiments with different patch sizes. For the following experiments, $\theta$ is set to 0.4 and the patching sizes we tried are $600 * 600$ and $1333 * 800$. SSD300 models were trained for 24 epochs using an SGD optimizer. The learning rate was set to $1 e-4$ at the start and changed at the 16th and 22nd iteration. The weight decay was set to $5 e-4$ and the momentum to 0.9 . Faster R-CNN or 
Cascade R-CNN models were trained for 12 epochs using an SGD optimizer with a learning rate of $2 e-3$, changing at the 8 th and 11th epochs, a momentum of 0.9 and a weight decay of $5 e-4$. All the results of these experiments are summarized in Table 2 . These

\begin{tabular}{|l|l|l|l|}
\hline architecture & patch size & mAP $(\%)$ & Recall $(\%)$ \\
\hline \multirow{2}{*}{ SSD300 } & no patches & 41.1 & 71.2 \\
\cline { 2 - 4 } & $600 * 600$ & 72.7 & 83.1 \\
\hline \multirow{3}{*}{ Faster R-CNN } & no patches & 79.3 & 89.7 \\
\cline { 2 - 4 } & $600 * 600$ & 78.6 & 89.9 \\
\cline { 2 - 4 } & $1333 * 800$ & 80.2 & 90.4 \\
\hline \multirow{3}{*}{ Cascade R-CNN } & no patches & 79.0 & 87.6 \\
\cline { 2 - 4 } & $600 * 600$ & 79.0 & 89.9 \\
\cline { 2 - 4 } & $1333 * 800$ & 80.3 & 90.1 \\
\hline
\end{tabular}

Table 2: Results of different architectures with different patch sizes (mAP and Recall)

results demonstrate that image patching method improves both mAP score and Recall. For example, in the case of SSD300, image patching method improves more than $30 \%$ in mAP with the patch size of $600 * 600$. On the other hand, the performance improvement is marginal, around 1\%, in the case of Faster R-CNN and Cascade $\mathrm{R}-\mathrm{CNN}$ with the patch size of $1333 * 800$. Similar results of Faster R-CNN and Cascade R-CNN an be explained by similarity of their architectures as explained in Section 3.2. Moreover, Figure 2 illustrates an example of face detection using Faster R-CNN with and without image patching. These images show that image patching leads to better detection results.

We recommend choosing the patch size as equivalent to the dimension of the detector's input layer, such as $1333 * 800$ for Faster $\mathrm{R}-\mathrm{CNN}$, because we will not lose information in the original artwork before passing it to the network. However, when the dimension of the input layer is small, such as $300 * 300$ for SSD300, faces are more likely to be divided into multiple patches, and the training does not work well. The patch size should be adapted relative to the size of the bounding box, and we found out that the patch size of $600 * 600$, which is the double size of the input layer, is more beneficial for SSD300. Hence if the patch size is too small for the size of bounding boxes, we choose multiples of the input layer size, but keep the original aspect ratio so that patches are not distorted. Based on the experimental results, we confirmed that the best choice is R-CNN, either Faster R-CNN or Cascade R-CNN, with size $1333 * 800$ image patching.

\subsection{Hyperparameter tuning for image patching}

Image patching method has an important hyperparameter $\theta$, which represents the threshold of IoU determining if a bounding box is contained in a patch or not, thereby affecting the creation of the training set containing faces. To evaluate the influence of $\theta$, we used Faster R-CNN with the patch size of $1333 * 800$, because it is the simplest of the two R-CNN architectures while yielding similar results with the more complex Cascade R-CNN. Four detectors were independently trained on the KaoKore dataset for 12 epochs, using an SGD optimizer (same parameters as in Section 4.2) for the

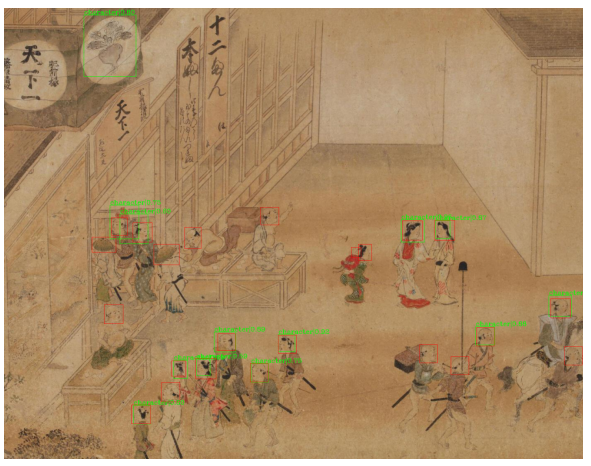

(a)

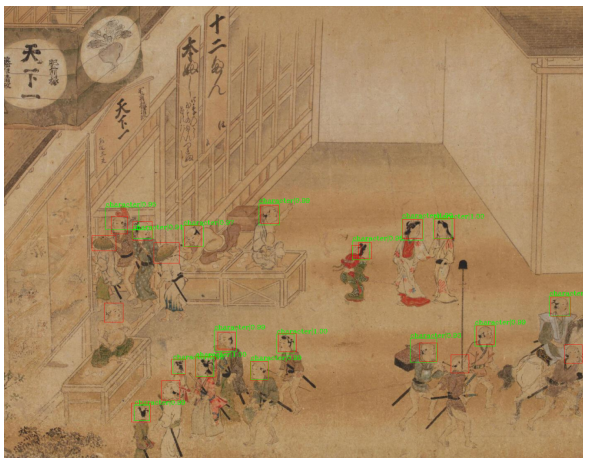

(b)

Figure 2: Comparison of two Faster R-CNN detectors, one trained with the image patching technique and the other without it, on the same artwork. On top, detection is done using the network without patches. Below, detection is done using the network with the patch size of $1333 * 800$. In green are the predicted bounding boxes and in red the ground truth bounding boxes. Source: "Kouhon Dataset" (owned by Shojo-Kouji)

different choices of $\theta$ between 0.4 and 0.7. Table 3 shows that the

\begin{tabular}{|l|l|l|l|l|l|}
\hline IoU threshold & 0.4 & 0.5 & 0.6 & 0.7 & No patching \\
\hline mAP $(\%)$ & 80.2 & 82.9 & 83.2 & 82.7 & 79.0 \\
\hline recall $(\%)$ & 90.4 & 91.1 & 90.9 & 90.5 & 89.7 \\
\hline
\end{tabular}

Table 3: Influence of the IoU threshold $\theta$ on the detection results of Faster R-CNN with the patch size of $1333 * 800$.

choice of the IoU threshold influences how a Faster R-CNN model achieves its detection task. Using the threshold of 0.4, we improved the detection score (mAP) with 1 points. Higher thresholds allow us to gain up to 4 points in precision. On the other hand, improvement is not significant in recall. This result suggests that the choice of the IoU threshold have a larger influence on precision than on recall. 


\section{FACE DETECTION FOR ART HISTORY STUDIES}

Our work is based on collaboration between computer scientists and art historians. Hence the final goal of our research is not only on improving the performance of the model on the standard dataset, but on applying our model to completely new datasets for assisting art historians annotate new datasets. Annotation for facial expressions is composed of two steps; (1) face detection for localization and (2) face interpretation for metadata. The goal of this paper is to automate the first step, and increase more time for art historians to focus on the second part. We call this whole process as semiautomatic annotation. A question here is if our model trained on the KaoKore dataset can be transferred to a new dataset that art historians need to work, and if the model actually reduce the time of the annotation workflow.

\subsection{Kouhon dataset}

The target of our experiment is the Kouhon dataset, which is another Japanese artworks from Yugyo Shounin Engi-Emaki Shojo-Kouji Kouhon collection (hereafter we call it 'Kouhon'), owned by ShojoKouji and digitized by Yugyoji Museum. Kouhon is a collection of picture scrolls in the 14th century with 346 sheets of paper in 10 volumes. Digitized images of this collection are not publicly available, and we obtained permission to use them for the purpose of research under the collaboration with the owner and the museum. This artwork is well known for many faces appearing across the scroll, so it is a daunting task for art historians to draw bounding boxes manually for all faces. Hence we applied our face detector to automate the detection of faces with coordinates of the bounding box.

For this task, we chose Faster R-CNN detector using the image patching method with $\theta$ set to 0.5 . We chose this detector because it was the one attaining the highest recall on the KaoKore dataset (Table 3). After the interview with art historians, they prefer higher recall than higher precision, because the cost of removing unnecessary faces that are detected are smaller than adding necessary faces that are not detected. The Kouhon dataset also contains text-only pages, but we did not remove those pages because they can be easily filtered out later. Figure 3 shows that our model achieves good results on the Kouhon dataset, suggesting that the model can generalize well to unseen data. The model did not detect many false positives on text-only pages (about one error per page) and blank pages. Those pages can be easily removed by human annotators beforehand, but even without filtering, we confirmed that pages without any faces do not cause the problem of false positives.

\subsection{Evaluation from art history studies}

The result of face detection on the Kouhon dataset was then evaluated by art historians. First, bounding box coordinates were visualized on the original images. Second, art historians can either (1) choose the bounding box as it is, (2) modify the bounding box in the neighborhood, (3) remove the bounding box, or (4) add a new bounding box. By repeating this process, art historians successfully created the complete annotation of faces in the Kouhon dataset with the help of automated face detection. After that, we evaluated

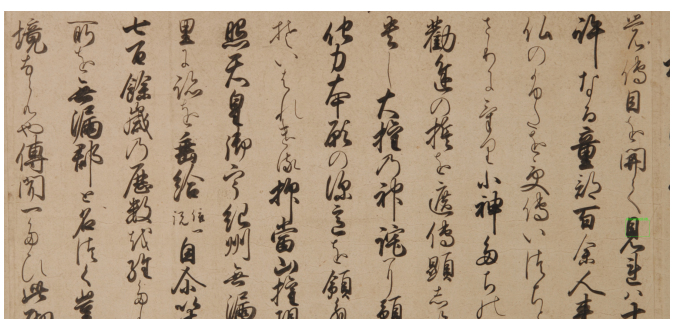

(a)



(b)



(c)

Figure 3: Example of detection using a trained Faster R-CNN with image patching with the patch size of $1333 * 800$ on the Kouhon dataset. In green are the predicted bounding boxes. Source: "Kouhon Dataset" (owned by Shojo-Kouji)

the complete annotation in terms of precision and recall to roughly evaluate how much time is reduced by automating face detection.

As a subjective evaluation by art historians, their impression was very positive. Our method not only reduced their effort for drawing rectangles, but also reduced mistakes by human errors. An objective evaluation also demonstrates the effectiveness of our approach.

(1) Least restrictive setup: precision of $85.99 \%$ and recall of $67.07 \%$ were achieved when we consider that every box found by our model that was nearly correct but had to be modified by experts was in fact correct.

(2) Moderately restrictive setup: precision of $85.94 \%$ and recall of $67.02 \%$ were achieved when we consider that nearly correct boxes were indeed correct when they had at least a Jaccard overlap of 0.5 with their ground truth counterparts.

(3) Most restrictive setup: precision of $84.17 \%$ and recall of $64.65 \%$ were achieved when we consider that Jaccard overlap of more than 0.9 is considered correct boxes. 
Finally, we estimate the reduction of cost measured in time for face detection within the annotation workflow. As described before, art historians had four choices on seeing the result of automated face detection. As the result of decision making on four choices, the time for annotation of the whole book can be represented as follows.

$$
\text { time }=t_{\text {acc }} * N_{\text {cor }}+t_{\text {mod }} * N_{\text {nearly-cor }}+t_{\text {rem }} * N_{\text {incorr }}+t_{\text {add }} * N_{\text {miss }}
$$

where $t_{\text {acc }}$ corresponds to the time to just accept the suggested bounding box, $t_{\text {mod }}$, to the time to modify a nearly correct bounding box, $t_{\text {rem }}$, to the time to remove a false positive bounding box, and $t_{\text {add }}$, to the time for a human to draw a new bounding box from scratch. Ns are the number of bounding boxes corresponding to each case.

To estimate those parameters, we made an interview on art historians. As a result, $t_{\mathrm{acc}}$ can be assumed to be negligible, because they do not have to do anything. $t_{\text {rem }}$ is also small, because it is easy to decide if the image is face or not. On the other hand, $t_{\text {mod }}$ needs a little time to edit, but it is still much smaller than $t_{\text {add }}$. Hence the main part of the equation is time $\approx t_{\text {add }} * N_{\text {miss }}$, where the ratio of $N_{\text {miss }}$ is equal to $1-$ recall. Because the result shows that recall is about $2 / 3$, we can conclude that the cost of annotation measured in time is reduced to almost $1 / 3$ in comparison to the fully manual workflow.

This result suggests the possibility of pursuing higher recall more aggressively by sacrificing precision, because higher recall may lead to less cost for annotation. This idea, however, does not work in reality for two reasons. First, lower precision means that art historians should see many bad bounding boxes on the result, and this will damage their trust in the machine learning system. The most annoying work for experts is to "fix errors caused by machines," which does not happen when humans work from the beginning. An acceptance level for precision may be difficult to measure, but at least precision should be reasonably high for the system to be accepted. Second, false negatives are actually caused by samples which are not in the training samples. As explained in Section 6, some false negative faces do not show any facial components, and the presence of faces are only inferred from other body components. In this case, lower precision does not automatically leads to higher recall. Hence the aggressive pursuit of recall is not a realistic choice.

\section{DISCUSSION}

First, we analyze the reason of better performance by image patching. We have two hypotheses. First, image patching works because it can keep higher image resolution and hence keep the detail of the input image. Second, image patching works because it can expand the effective size of the training set. We first discuss the first hypothesis. Figure 4) compares the result of with or without image patching in the training phase. Even when the image patching was not used in the training phase, image patching in the detection phase can increase the quality of the result. This result indicates that having higher image resolution input is always beneficial for a face detector. We next discuss the second hypothesis. Because image patching splits the original images into five patches, the effective size of the training set can be increased if we consider each patch as a new training set. For example, from 1137 images and 6692 faces in the original training set, image patching with the patch size of $1333 * 800$ generates 4618 positive samples containing 13453 faces in Intra-books split. By doubling the size of the training set, face detectors can take advantage of more training data, which is especially important for a small dataset as the KaoKore dataset. Based on the above discussion, we conclude that both higher image resolution and dataset expansion contributed to better performance of image patching.

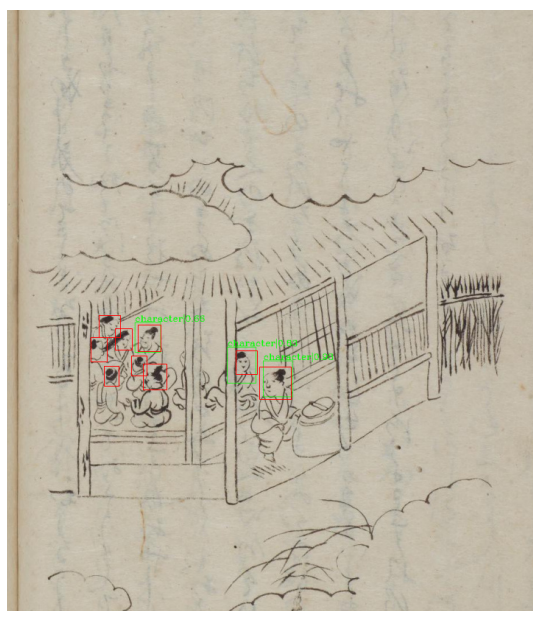

(a)

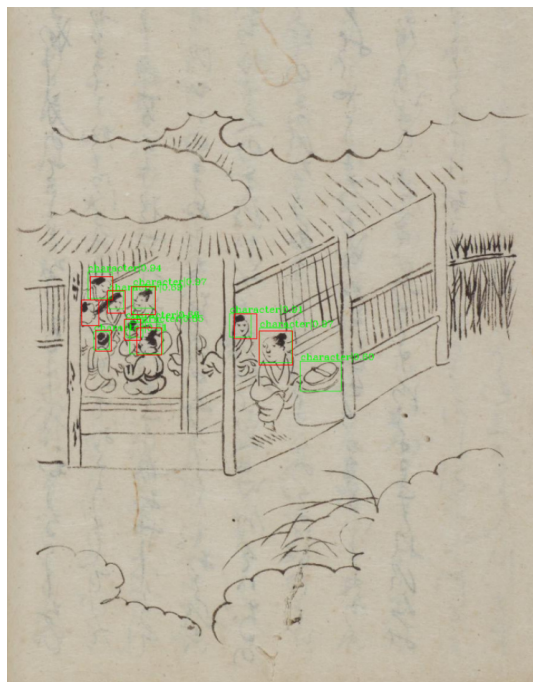

(b)

Figure 4: The same Faster $\mathrm{R}-\mathrm{CNN}$ is used to detect faces on the same image in two different setups. The top and the bottom image is the result without image patching and with image patching, respectively. Source: "Kouhon Dataset" (owned by Shojo-Kouji)

Second, false negatives in our experiments are partly due to the high level interpretation of artworks by art historians. For example, Figure 5 contains both the detection outputs from our model and the 
curated version of the output by art historians. In the considered artwork, we can see that some of the faces are fully hidden by veils and hats, so it is actually difficult for our network to detect them as faces. On the other hand, the human annotator, using the knowledge that these veils must hide a face, can crop them as faces. For our network to be able to have the same reasoning, we should train our network on such faces. On the other hand, this retraining could lead to an increase in false positives since these hidden faces are not sharing lots of graphical features with the ones that are not hidden. These examples suggest the limitation of automated face detection in terms of unseen types of faces and the requirement of high-level semantic interpretations. It is always important to design a system for experts to modify the output of automated systems.

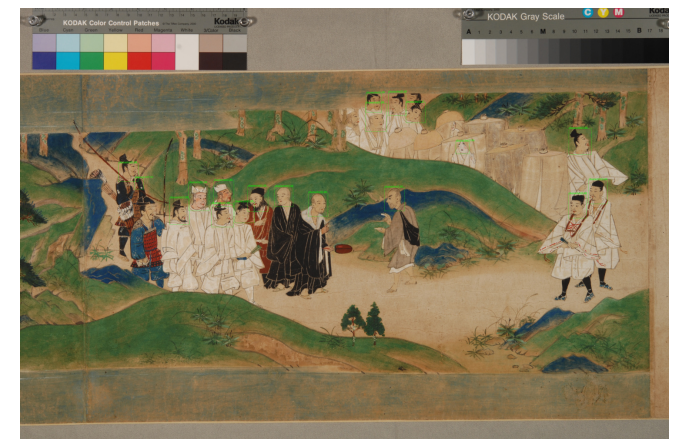

(a)

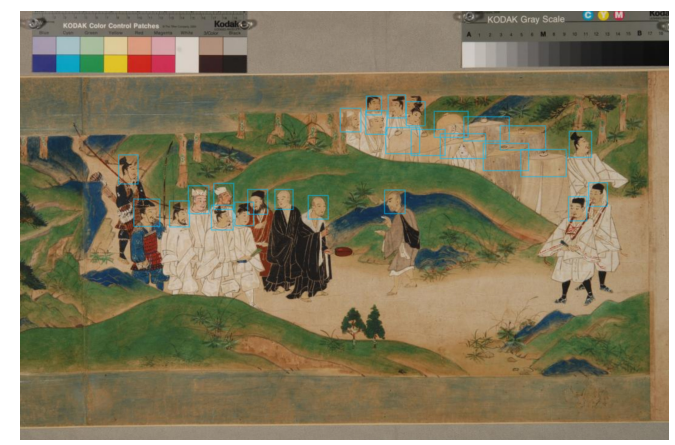

(b)

Figure 5: The top image shows the result of automatic face detection. The bottom image shows the result of annotation by art historians based on the output of automatic face detection. Source: "Kouhon Dataset" (owned by Shojo-Kouji)

As described before, the whole annotation workflow consists of detection and interpretation tasks, so a natural question is if we can also automate the interpretation tasks. The KaoKore dataset has manually annotated metadata for each face, describing such as social status in the pre-modern Japanese society, a genre and an orientation. Using these labels, the original paper of the KaoKore dataset[15] summarized the baseline performance of genre classifiers. Following this work, we also designed a classifier for the status of faces using the ResNext architecture[17], and the result shows that the precision reached $85.009 \%$. We also applied GradCAM and other visualization tools for feature space to understand which features are key to classify different classes. We discussed those results with art historians and realized that current tools are not explainable and hence not understandable for them. As a result, we regard this task as future work.

This problem is also related to how the classification task should be defined for art history research, where interpretation itself evolves over time as we understand more deeply about artworks, and the definition of the class is reflecting the level of understanding. To avoid this complexity, we focused on the detection task in this paper, and at least we showed that the proposed tool has value for humanities scholars. The future work includes not only a machine learning model that does both detection and classification at the same time, but also focus more on the measurement of facial features $[10,18]$.

\section{CONCLUSION}

This paper proposed a face detection method for pre-modern Japanese artworks using R-CNN and image patching. We showed the effectiveness of image patching in terms of higher image resolution and dataset expansion, which is beneficial for R-CNN-based object detectors. We first used the KaoKore dataset to design our face detector, and then applied our model to the Kouhon dataset to demonstrate the impact of the model to art history research, such as reducing the time for annotation to $1 / 3$ in comparison to fully manual workflow. As the next step, fully automated annotation workflow doing both the detection and labelling tasks at the same time, as discussed in section 6 , is a challenging theme in addition to the fine-tuning of the model and taking recent developments in object detection research.

The code we used in the paper will be released to the public in the future. To test the code, the KaoKore dataset can be used as open data, but the Kouhon dataset is closed within the research group.

\section{ACKNOWLEDGMENTS}

This work was mainly performed while the first author was supported by the NII internship program. KaoKore dataset is created by ROIS-DS Center for Open Data in the Humanities from Japanese artwork images released from National Institute of Japanese Literature, Keio University Media Center, and Kyoto University Library Network. Special appreciation goes to Shojo-Kouji and Yugyoji Museum for allowing us to use the Kouhon dataset for collaborative research.

\section{REFERENCES}

[1] R. Bai, H. Ling, Z. Kai, D. Qi, and Q. Wang. 2019. Author recognition of Fine-Art paintings. In 2019 Chinese Control Conference (CCC). 8513-8518.

[2] Zhaowei Cai and Nuno Vasconcelos. 2017. Cascade R-CNN: Delving into High Quality Object Detection. arXiv:1712.00726 [cs.CV]

[3] Kai Chen, Jiaqi Wang, Jiangmiao Pang, Yuhang Cao, Yu Xiong, Xiaoxiao Li, Shuyang Sun, Wansen Feng, Ziwei Liu, Jiarui Xu, Zheng Zhang, Dazhi Cheng, Chenchen Zhu, Tianheng Cheng, Qijie Zhao, Buyu Li, Xin Lu, Rui Zhu, Yue Wu, Jifeng Dai, Jingdong Wang, Jianping Shi, Wanli Ouyang, Chen Change Loy, and Dahua Lin. 2019. MMDetection: Open MMLab Detection Toolbox and Benchmark. arXiv preprint arXiv:1906.07155 (2019).

[4] Ahmed Elgammal, Marian Mazzone, Bingchen Liu, Diana Kim, and Mohamed Elhoseiny. 2018. The Shape of Art History in the Eyes of the Machine. arXiv:1801.07729 [cs.AI] 
[5] Kaiming He, Xiangyu Zhang, Shaoqing Ren, and Jian Sun. 2015. Deep Residual Learning for Image Recognition. arXiv:1512.03385 [cs.CV]

[6] Wei Liu, Dragomir Anguelov, Dumitru Erhan, Christian Szegedy, Scott Reed, Cheng-Yang Fu, and Alexander C. Berg. 2016. SSD: Single Shot MultiBox Detector. Lecture Notes in Computer Science (2016), 21-37. https://doi.org/10.1007/978-3319-46448-0_2

[7] Prathmesh Madhu, Ronak Kosti, Lara Mührenberg, Peter Bell, Andreas Maier and Vincent Christlein. 2019. Recognizing Characters in Art History Using Deep Learning. In Proceedings of the 1st Workshop on Structuring and Understanding of Multimedia HeritAge Contents (Nice, France) (SUMAC '19). Association for Computing Machinery, New York, NY, USA, 15-22. https://doi.org/10.1145/ 3347317.3357242

[8] Toru Ogawa, Atsushi Otsubo, Rei Narita, Yusuke Matsui, Toshihiko Yamasaki, and Kiyoharu Aizawa. 2018. Object Detection for Comics using Manga109 Annotations. arXiv: 1803.08670 [cs.CV]

[9] Shaoqing Ren, Kaiming He, Ross Girshick, and Jian Sun. 2015. Faster RCNN: Towards Real-Time Object Detection with Region Proposal Networks. arXiv:1506.01497 [cs.CV]

[10] B. Renoust, M.O. Franca, J. Chan, N. Garcia, V. Le, A. Uesaka, Y. Nakashima, H. Nagahara, J. Wang, and Y. Fujioka. 2019. Historical and modern features for Buddha statue classification. In SUMAC 2019 - Proceedings of the 1st Workshop on Structuring and Understanding of Multimedia heritAge Contents, co-located with MM 2019. https://doi.org/10.1145/3347317.3357239
[11] C. Sandoval, E. Pirogova, and M. Lech. 2019. Two-Stage Deep Learning Approach to the Classification of Fine-Art Paintings. IEEE Access 7 (2019), 41770-41781.

[12] Karen Simonyan and Andrew Zisserman. 2014. Very Deep Convolutional Networks for Large-Scale Image Recognition. arXiv:1409.1556 [cs.CV]

[13] C. Sirirattanapol, Y. Matsui, S. Satoh, K. Matsuda, and K. Yamamoto. 2017. Deep Image Retrieval Applied on Kotenseki Ancient Japanese Literature. In 2017 IEEE International Symposium on Multimedia (ISM). 495-499.

[14] S. Smirnov and A. Eguizabal. 2018. Deep learning for object detection in fine-art paintings. In 2018 Metrology for Archaeology and Cultural Heritage (MetroArchaeo). $45-49$.

[15] Yingtao Tian, Chikahiko Suzuki, Tarin Clanuwat, Mikel Bober-Irizar, Alex Lamb, and Asanobu Kitamoto. 2020. KaoKore: A Pre-modern Japanese Art Facial Expression Dataset. arXiv:2002.08595 [cs.CV]

[16] Nicholas Westlake, Hongping Cai, and Peter Hall. 2016. Detecting People in Artwork with CNNs. In European Conference on Computer Vision. Springer, 825841.

[17] Saining Xie, Ross Girshick, Piotr Dollár, Zhuowen Tu, and Kaiming He. 2016. Aggregated Residual Transformations for Deep Neural Networks. arXiv:1611.05431 [cs.CV]

[18] Jordan Yaniv, Yael Newman, and Ariel Shamir. 2019. The Face of Art: Landmark Detection and Geometric Style in Portraits. ACM Trans. Graph. 38, 4, Article 60 (July 2019), 15 pages. https://doi.org/10.1145/3306346.3322984 